\title{
An Inherited Cause of Stroke Mimic in a Toddler
}

\author{
Sangeetha Yoganathan ${ }^{1} \cdot{\text { Madhan } K \text { Mmar }^{2} \cdot \text { Lenka Sushma }^{1} \cdot \text { Sumita Danda }}^{3} \cdot$ Maya Thomas $^{1}$
}

Received: 23 June 2021 / Accepted: 15 July 2021 / Published online: 1 September 2021

(c) Dr. K C Chaudhuri Foundation 2021

To the Editor: Stroke mimic is one of the common causes of neurological deficits in young children. A 2-y-old-boy born to nonconsanguineous parents presented with recurrent weakness of left upper and lower limbs alternating with right upper and lower limbs, each event lasting for 48-72 h. The child was delivered at $36 \mathrm{wk}$ of gestation with a birth weight of $2130 \mathrm{~g}$. He had daily episodes of abnormal twisting posture of limbs and trunk with preserved awareness from first month of age. Mild developmental delay was observed. Left eye convergent squint and right hemiplegia were observed. Possibilities of alternating hemiplegia of child (AHC), mitochondrial encephalomyopathy with lactic acidosis and stroke (MELAS) and Moyamoya syndrome were considered. Brain magnetic resonance imaging with magnetic resonance angiography and electroencephalography were normal. Next generation sequencing revealed a likely pathogenic heterozygous missense variation in the exon 17 of ATPIA3 gene (c.2440G >A; p.Asp814Asn; ENST00000545399.6) confirming the diagnosis of alternating hemiplegia of childhood-2.

AHC is a rare inherited cause of stroke mimic in infants and children. The key differentiating features of stroke mimics from stroke are gradual onset symptoms, presence of altered sensorium, nonvascular territory involvement, absence of vascular risk factors, absence of ischemic or hemorrhagic lesions on neuroimaging, and rapid recovery [1]. ATP1A3related neurological disorders include rapid onset dystoniaparkinsonism (RDP), AHC and cerebellar ataxia, areflexia, pes cavus, optic atrophy, and sensorineural deafness (CAPOS) [2]. AHC typically has onset before 18 mo with repeated episodes of neurological symptoms of variable duration. Neurological symptoms include alternating hemiparesis,

Maya Thomas

maya@cmcvellore.ac.in

1 Department of Neurological Sciences, Christian Medical College, Vellore-632004, Tamil Nadu, India

2 Department of Pediatrics, Christian Medical College, Vellore- 632004, Tamil Nadu, India

3 Department of Medical Genetics, Christian Medical College, Vellore- 632004, Tamil Nadu, India quadriparesis, dystonic spells, oculomotor abnormalities, developmental delay, ataxia and autonomic symptoms [3]. Our patient had developmental delay, paroxysms of dystonia, and alternating hemiparesis. The pathophysiological mechanisms described in ATP1A3-related neurological disorders include dysfunctional neuronal sodium-potassium ATPase pump and imbalance of glutaminergic-GABAergic activity [4]. Treatment includes avoidance of sleep deprivation and stress, flunarizine, topiramate, benzodiazepines, steroid, amantadine, memantine, aripiprazole, adenosine triphosphate, coenzyme Q, acetazolamide, dextromethorphan, ketogenic diet and vagus nerve stimulation [4].

\section{Declarations}

Conflict of Interest None.

\section{References}

1. Buck BH, Akhtar N, Alrohimi A, Khan K, Shuaib A. Stroke mimics: incidence, aetiology, clinical features and treatment. Ann Med. 2021;53:420-36.

2. Brashear A, Sweadner KJ, Cook JF, et al. ATP1A3-Related Neurologic Disorders. 2008 Feb 7 [Updated 2018 Feb 22]. In: Adam MP, Ardinger HH, Pagon RA, et al., editors. GeneReviews®. Seattle (WA): University of Washington, Seattle; 1993-2021. Available at: https://www.ncbi.nlm.nih.gov/books/NBK1115/. Accessed on 17 Jun 2021

3. Rosewich H, Sweney MT, DeBrosse S, et al. Research conference summary from the 2014 international task force on ATP1A3 -related disorders. Neurol Genet. 2017;3:e139.

4. Samanta D. Management of alternating hemiplegia of childhood: a review. Pediatr Neurol. 2020;103:12-20.

Publisher's Note Springer Nature remains neutral with regard to jurisdictional claims in published maps and institutional affiliations. 